\title{
A Differential Approach to Shape from Polarization
}

\author{
Roberto Mecca ${ }^{1,2}$ \\ rm822@cam.ac.uk \\ Fotios Logothetis ${ }^{1}$ \\ fl302@cam.ac.uk \\ Roberto Cipolla ${ }^{1}$ \\ rc10001@cam.ac.uk
}

${ }^{1}$ Department of Engineering, University of Cambridge, UK

${ }^{2}$ Department of Mathematics, University of Bologna, Italy

\begin{abstract}
State-of-the-art formulations of the Shape from Polarisation problem consist of several steps based on merging physical principles that prevent this problem being described by a single mathematical framework. In addition, specular and diffuse reflections need to be separately considered, making the three-dimensional shape reconstruction not easily applicable to heterogeneous scenes consisting of different materials.

In this work we derive a unified specular/diffuse reflection parametrisation of the Shape from Polarisation problem based on a linear partial differential equation capable of recovering the level-set of the surface. The inherent ambiguity of the Shape from Polarization problem becomes evident through the impossibility of reconstructing the whole surface with this differential approach. To overcome this limitation, we consider shading information elegantly embedding this new formulation into a two-lights calibrated photometric stereo approach. Thus we derive an albedo independent and well-posed differential model based on a system of hyperbolic PDEs capable of reconstructing the shape with no ambiguity.

We validate the geometrical properties of the new differential model for the Shape from Polarisation problem using synthetic and real data by computing the isocontours of the shape under observation. Lastly, we show the suitability of this new model to elegantly fit into a variational solver that is able to provide 3D shape reconstructions from synthetic and real data.
\end{abstract}

\section{Acknowledgments}

Roberto Mecca was supported through a Marie Curie fellowship of the "Istituto Nazionale di Alta Matematica", Italy.

\section{Introduction}

Retrieving 3D shape features from polarisation cues acquired from a single point of view is a concept introduced by Koshikawa [ $\square]$ to constrain the surface normals of objects made out of dielectric materials. Together with the increasing impact of the Computer Vision for 3D scanning techniques, the so-called Shape from Polarisation problem (SfP) became one of the most physically based approaches in the Shape from X family. It is based on the physical property that unpolarised light becomes partially polarized once it reflects off an object. The acquisition process consists of taking images of a static object under the same illumination conditions, with a linear polariser in front of a camera. Each image differs from the others by the fact that the polariser has been rotated by a known angle from its initial position. What 
makes the SfP approach very interesting, at least in theory, is that polarised image formation is albedo and lighting independent. However, despite the theoretical and practical progress achieved over almost forty years of research, the SfP still provides limited constraints of the surface preventing the shape recovery (due to the periodicity of the polarisation information). Indeed, most existing SfP approaches usually combine other cues coming from multi-view

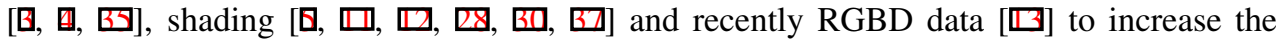
information of the 3D shape and fully reconstruct the surface under observation.

Furthermore, another important limitation of the SfP is its mathematical formulation. Instead of being expressed within a single framework, it is fragmented into several steps derived from physical models that describe the behaviour of light when it propagates between media of differing refractive indices [ $\square]$. This makes the SfP less straightforward to understand and less practical to solve than other Shape from X approaches.

Contribution The main contribution of this paper is to introduce the first differential formulation of the SfP through a linear PDE.

The importance of this formulation is threefold:

- It is albedo and reflection type (diffuse/specular) independent;

- It elegantly circumvents the SfP impossibility to recover the shape by providing isocontours of the surface;

- It can be easily used for merging SfP with other techniques having a differential formulation. In this work we enhance the SfP with two-lights shading information.

\section{Previous Works}

Polarimetric cues have been used in Computer Vision for a number of different tasks mostly related to the difficulty of dealing with specular highlights. In addition to using polarised

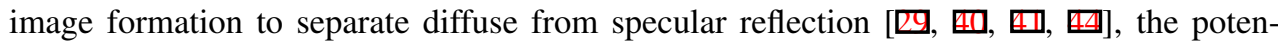
tial of polarimetric approaches has been demonstrated by its use in determining the normal orientation of glossy surfaces [ㄴ, 4 , 4 , 四]. For the same reason, particular attention has been given to the SfP problem attempting to recover the shape of transparent objects

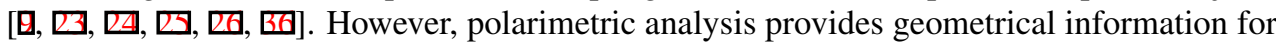
dielectric surfaces, which has allowed the SfP to be adopted to reconstruct surfaces reflecting light diffusively too [D].

In any case, the features of the surface provided by the polarization reflectance model are limited and this results to ambiguous shape recovery. To disambiguate polarisation normals, most of the approaches enhance the SfP with supplementary cues to make the overall methodology well-posed. Several works use multiple views of the object together with polarisation imaging $[\square, \square]$. Other approaches are more related to what we propose here, where shading cues are merged with polarisation imaging. For example, Drbohlav and Sara [ए] employed polarisation imaging to recover the zenith angle of the surface normal adding integrability constraints to reduce the ambiguity of the uncalibrated photometric stereo to concave/convex case. Morel et al. [ [ $[\mathbb{Z}]$ used a lighting system in a diffuse dome composed of a ring with numerous LEDs. It provides a uniform and unpolarized light onto the object to be digitalized. The object is placed inside the dome and the light reflected by its surface is analysed by the camera and the liquid crystal polariser. The ring of LEDs is split into four parts that can be independently electrically controlled. Huynh et al. [ $\square$ ] proposed an iterative method where diffuse polarisation modelling is considered together with two additional 
constraints of the problem, including the surface integrability and the material dispersion equation using a hyperspectral imaging system. They adopted a preliminary disambiguation proposed by Zhu and Shi [0] extended by the use of fast marching and patch stitching. Atkinson and Hancock [ब] disambiguated the polarisation normal using the shading information of three distant light sources. They are placed in a strategic position, such that the angles subtended by the camera and the light sources from the object are equal and that the distances between the object and the light sources are equal, too. Ngo et al. [B] proposed a very interesting approach based on the ratio of both lambertian irradiance equations (with uniform light direction) and polarisation image formation equations to compute the surface orientation and the refractive index using at least 3 light sources. By increasing the number of input images (i.e. light sources), they eventually extended the approach to the uncalibrated case, also computing the light directions.

Lastly, Smith et al. [ $[\mathrm{B}]$ ] proposed a SfP approach aided by shading information provided by a distant light source with orthographic viewing geometry. Besides estimating the shape up to a global concave/convex ambiguity, an important limitation for real applications is the assumption of uniform albedo.

Differently from all previous works, we present the first attempt of describing the SfP through a differential formulation, where specular and diffuse reflection in polarisation imaging are unified into a unique mathematical framework consisting of a homogeneous liner PDE. This brings a twofold advantage: to conceive the expected ambiguity of the SfP problem by extracting the level-set of the surface, and to embed such PDE into a differential formulation, modelling the Photometric Stereo problem using image ratios $[\boldsymbol{\square}, \mathbb{\square}, \mathbb{\square}, \mathbb{\square}$, [प], ㅁ], []].

Indeed, our approach considers shading information coming from a minimum of two point light sources of known position. The ratio of the respective irradiance equations leads to an albedo invariant PDE that ambiguously describes the surface [ $[\mathrm{U}]$. The combination of the two into a differential system makes the problem solvable.

\section{Shape from Polarization: a differential approach}

In this section, we describe the mathematical derivation of a novel framework for the SfP problem consisting of a homogeneous linear partial differential equation. To do so, before recalling the theoretical principles for polarised imaging, we firstly consider an important aspect of the surface normal parametrisation as a function of the depth.

\subsection{Camera parametrisation}

The pinhole camera modeling is an important aspect to consider while carefully taking into account image based shape reconstruction algorithms. When perspective viewing geometry comes into play, there have been several works proposing different parameterisations $[\mathrm{G}, \mathrm{B}, \mathrm{B}, \mathbf{B}]$ ]. However, to mathematically describe the SfP, we do not use any specific parameterization of the camera since we only consider a general aspect occurring when surface normal deforms due to perspective viewing geometry. For this purpose, let us call as $\chi(\mathbf{x}) \in \Sigma, z(\mathbf{x})$ and $\nabla z(\mathbf{x})=\left(z_{x}(\mathbf{x}), z_{y}(\mathbf{x})\right)$ the point belonging to the surface, the depth and the gradient of the surface at pixel $\mathbf{x}=(x, y)$. Then, the first two components of the non-unit normal vector to the surface $\overline{\mathbf{n}}(\mathbf{x})=\left(\bar{n}^{1}(\mathbf{x}), \bar{n}^{2}(\mathbf{x}), \bar{n}^{3}(\mathbf{x})\right)$ are proportional to $\nabla z(\mathbf{x})$ up to a factor depending on the focal length $f$. 
This means that, if we take the unit surface normal $\mathbf{n}=\frac{\overline{\mathbf{n}}}{\|\overline{\mathbf{n}}\|}$ into account, we have

$$
n^{1}(\mathbf{x})=g(f) \frac{z_{x}(\mathbf{x})}{\|\overline{\mathbf{n}}\|} \quad \text { and } \quad n^{2}(\mathbf{x})=g(f) \frac{z_{y}(\mathbf{x})}{\|\overline{\mathbf{n}}\|} .
$$

For completeness, let us mention that for the orthographic viewing geometry, (1) is still preserved as $g(f)=1$. In the following part, we use this fact to derive our new model independently from the camera viewing geometry.

\subsection{Polarization Imaging}

When a linear polariser filter is imposed front of the camera, the intensity of the light acquired by the sensor depends on the rotation angle of the polariser $\theta_{p o l}$. This makes the image formation model being per pixel as follows

$$
I\left(\theta_{\text {pol }}\right)=\frac{I_{\text {max }}+I_{\text {min }}}{2}+\frac{I_{\text {max }}-I_{\text {min }}}{2} \cos \left(2 \theta_{\text {pol }}-2 \bar{\theta}\right)
$$

where $\bar{\theta}$ is the phase angle. It is the angle that the linear polariser has to have in order to obtain the highest intensity $I_{\max }=I\left(\theta_{\text {pol }}=\bar{\theta}\right)$. Instead, $I_{\min }$ is the minimum intensity value obtainable while rotating the polariser. To simplify the notation, in the following we refer as $I_{+}=\frac{I_{\max }+I_{\min }}{2}$ and $I_{-}=\frac{I_{\max }-I_{\min }}{2}$.

Now, to introduce the depth parameter $z(\mathbf{x})$ in the polarisation image formation (2), we consider the parameterisation with spherical coordinates for the normalised version of the surface normal as

$$
\mathbf{n}(\mathbf{x})=\frac{\overline{\mathbf{n}}(\mathbf{x})}{\|\overline{\mathbf{n}}(\mathbf{x})\|}=(\cos (\theta) \sin (\phi), \sin (\theta) \sin (\phi), \cos (\phi))
$$

where $\theta \in[0,2 \pi]$ is the azimuth angle and $\phi \in\left[0, \frac{\pi}{2}\right]$ is the zenith angle. Let us recall that the phase angle $\bar{\theta}$ in (2) contains geometrical information regarding the shape since $\theta=\bar{\theta}$ or $\theta=\bar{\theta}+\pi$ that resumes the ambiguity of the SfP problem.

With the aim of deriving a differential formulation of the SfP, we introduce the depth parameters $z_{x}(\mathbf{x})$ and $z_{y}(\mathbf{x})$ in the image formation (2) by substituting (1) in the first two coordinates of (3), so we can get respectively the following equalities

$$
\cos (\theta)=g(f) \frac{z_{x}}{\|\overline{\mathbf{n}}(\mathbf{x})\| \sin (\phi)} \quad \text { and } \quad \sin (\theta)=g(f) \frac{z_{y}}{\|\overline{\mathbf{n}}(\mathbf{x})\| \sin (\phi)} .
$$

\subsection{The differential model for the Shape from Polarisation}

Let us rearrange the image formation model (2) using the following trigonometric formula

$$
\cos \left(2 \theta_{p o l}-2 \bar{\theta}\right)=\cos \left(2 \theta_{p o l}\right) \cos (2 \bar{\theta})+\sin \left(2 \theta_{p o l}\right) \sin (2 \bar{\theta})
$$

and the duplication formulas which lead to the following equality

$$
\cos \left(2 \theta_{p o l}-2 \bar{\theta}\right)=\cos \left(2 \theta_{p o l}\right)\left(2 \cos ^{2}(\bar{\theta})-1\right)+2 \sin \left(2 \theta_{p o l}\right) \sin (\bar{\theta}) \cos (\bar{\theta}) .
$$

We proceed by considering two angles of polarization 0 and $\frac{\pi}{4}$ that make (6) into the following equations

$$
\cos (-2 \bar{\theta})=2 \cos ^{2}(\bar{\theta})-1 \quad \text { and } \quad \cos \left(\frac{\pi}{2}-2 \bar{\theta}\right)=2 \sin (\bar{\theta}) \cos (\bar{\theta})
$$


which lead to the following image formation equations

$$
\left\{\begin{array} { l } 
{ I _ { 0 } = I _ { + } + I _ { - } ( 2 \operatorname { c o s } ^ { 2 } ( \overline { \theta } ) - 1 ) } \\
{ I _ { \frac { \pi } { 4 } } = I _ { + } + I _ { - } ( 2 \operatorname { s i n } ( \overline { \theta } ) \operatorname { c o s } ( \overline { \theta } ) ) }
\end{array} \quad \stackrel { \text { by substituting } ( 4 ) } { \longrightarrow } \quad \left\{\begin{array}{l}
I_{0}=I_{+}+I_{-}\left(2 g^{2}(f) \frac{z_{x}^{2}}{\|\overline{\mathbf{n}}\|^{2} \sin ^{2}(\phi)}-1\right) \\
I_{\frac{\pi}{4}}=I_{+}+I_{-}\left(2 g^{2}(f) \frac{z_{x} z_{y}}{\|\overline{\mathbf{n}}\|^{2} \sin ^{2}(\phi)}\right)
\end{array}\right.\right.
$$

where we used the consistency of shape information from the polarisation image formation angle $\bar{\theta}$ with respect to the surface normal. After some algebra we get

$$
\begin{aligned}
& 2 I_{-} g^{2}(f) \frac{z_{x}^{2}}{\|\overline{\mathbf{n}}\|^{2} \sin ^{2}(\phi)}=I_{0}-I_{+}+I_{-} \\
& 2 I_{-} g^{2}(f) \frac{z_{x} z_{y}}{\|\overline{\mathbf{n}}\|^{2} \sin ^{2}(\phi)}=I_{\frac{\pi}{4}}-I_{+} .
\end{aligned}
$$

With the aim of eliminating the dependency on the zenith angle $\phi$ and the non-linear part due to $\|\overline{\mathbf{n}}\|$, we consider the ratio between (9) and (10) getting the following linear homogeneous PDE

$$
z_{x}\left(-I_{\frac{\pi}{4}}+I_{+}\right)+z_{y}\left(I_{0}-I_{+}+I_{-}\right)=0 .
$$

As a first remark, we notice that (11) is invariant with respect to lighting and albedo. Most importantly, it describes the geometry of the surface through its isocontours circumventing the ambiguity of the SfP problem.

In the next section we describe how (11) elegantly fits into a well-posed differential system of hyperbolic PDEs completing the ill posed Shape from Photometric Stereo with only two light sources [B]].

\section{Enhancing SfP with two-lights Photometric Stereo}

In this section we describe how to exploit the new differential formulation for the SfP (11) into a well-posed framework where shading information is provided.

In order to take advantage of the fact that SfP is albedo independent, we consider a fully calibrated Photometric Stereo (SfPS) approach having the same specific feature by basing the derivation on the irradiance equations ratio [四]. Unlike polarisation theory, in order to parametrize the SfPS approach, we require some additional information to be know: the camera parameters, the lighting and the type of reflection (i.e. diffuse or specular).

Let us consider the approach proposed by Mecca et al. [미 which uses the camera modeling introduced by Papadhimitri and Favaro [B]

$$
\overline{\mathbf{n}}(\mathbf{x})=\frac{1}{f}(f \nabla z(\mathbf{x}),-f-z(\mathbf{x})-\mathbf{x} \cdot \nabla z(\mathbf{x}))
$$

and it is based on the following irradiance equation

$$
I_{i}(\mathbf{x})=\rho(\mathbf{x}) a_{i}(\mathbf{x}, z)\left(\mathbf{n}(\mathbf{x}) \cdot \mathbf{h}_{i}\left(\mathbf{l}_{i}(\mathbf{x}, z), \mathbf{v}(\mathbf{x}, z)\right)\right)^{\frac{1}{c(\mathbf{x})}} \quad \text { where } \quad i=1,2 .
$$

Although (13) is not physically based, it describes the mixture of reflections in a single equation instead of considering the linear combination of diffuse and specular reflection [G]. Indeed, the single reflection lobe changes size dynamically, depending on the specular parameter $c(\mathbf{x})$ and the half vector between the light $\mathbf{l}_{i}(\mathbf{x}, z)$ and the viewer direction $\mathbf{v}(\mathbf{x}, z)$ defined as follows 


$$
\mathbf{h}_{i}(\mathbf{x}, z)=\left(h_{i}^{1}(\mathbf{x}, z), h_{i}^{2}(\mathbf{x}, z), h_{i}^{3}(\mathbf{x}, z)\right)=\overline{\mathbf{l}}_{i}(\mathbf{x}, z)+\min \left\{1, \frac{|1-c(\mathbf{x})|}{\varepsilon}\right\} \overline{\mathbf{v}}(\mathbf{x}, z)
$$

where $\varepsilon$ (assumed equal to 0.01 for the experiments) defines a transition phase that averages diffuse and specular reflection.

We assume that the light spreads according to the point light source parametrisation at point $\mathbf{P}_{i}(\mathbf{x})$ and attenuation $a_{i}(\mathbf{x}, z)$ as follows

$$
\mathbf{l}_{i}(\mathbf{x}, z)=\chi(\mathbf{x})-\mathbf{P}_{i}(\mathbf{x}) \quad \text { and } \quad a_{i}(x, y, z)=\frac{\phi_{i}\left(\mathbf{l}_{i}(\mathbf{x}, z) \cdot \mathbf{p}_{i}\right)^{v}}{\left|\overline{\mathbf{l}}_{i}(x, y, z)\right|^{2}}
$$

where $\mathbf{p}_{i}$ is the main direction of illumination that we assume equal to $(0,0,1), v$ is the coefficient of radial attenuation and $\phi_{i}$ is the intensity of the $i^{t h}$ point light source. For our experiments, we consider all these quantities as known.

Finally, the unknown albedo $\rho(\mathbf{x})$ cancels out by considering the ratio $\frac{I_{1}(\mathbf{x})}{I_{2}(\mathbf{x})}$ that yields the following quasi-linear PDE

$$
\mathbf{b}_{p s}(\mathbf{x}, z) \cdot \nabla z(\mathbf{x})=s_{p s}(\mathbf{x}, z)
$$

where, by dropping the dependency on $\mathbf{x}$ and $z$, we have

$$
\begin{gathered}
\mathbf{b}_{p s}=\left(\left(\phi_{1} a_{2} I_{1}\right)^{c}\left(f h_{2}^{1}-x h_{2}^{3}\right)-\left(\phi_{1} a_{1} I_{2}\right)^{c}\left(f h_{1}^{1}-x h_{1}^{3}\right),\left(\phi_{2} a_{2} I_{1}\right)^{c}\left(f h_{2}^{2}-y h_{2}^{3}\right)-\left(\phi_{1} a_{1} I_{2}\right)^{c}\left(f h_{1}^{2}-y h_{1}^{3}\right)\right) \\
s_{p s}=(f+z)\left(\left(\phi_{2} a_{2} I_{1}\right)^{c} h_{2}^{3}-\left(\phi_{1} a_{1} I_{2}\right)^{c} h_{1}^{3}\right) .
\end{gathered}
$$

Resuming, the system of PDEs consisting of (11) and (16) counts the minimum amount of equations to have the SfP and SfPS problem unified under a single and well-posed differential framework. Due to lack of space we do not provide mathematical details about that. We resume the basic idea for which the solvability of this system is given by the fact that the characteristic lines of each hyperbolic equation are not parallel. This allows to propagate the information starting from a single point towards the whole image domain by linearly combining the two linearly independent characteristic fields [미].

\subsection{Numerical approach to the SfP + SfPS}

In this section, to make the new differential model for the SfP more consistent with the SfPS one (16), we consider (11) in the form $\mathbf{b}_{\text {pol }}(\mathbf{x}) \cdot \nabla z(\mathbf{x})=0$.

For computing the sinusoid given by the parameters $\left(I_{+}, I_{-}, \bar{\theta}\right)$, we avoid the standard procedure taking only the images $I_{0}, I_{\frac{\pi}{4}}$ and $I_{\frac{\pi}{2}}[\mathrm{H}]$ since it performs poorly in practice, as differences between different polarisation images are very small and hence sensitive to noise.

With the aim to maximise robustness to noise, we capture several images $I_{1} \ldots I_{n}$ at polarisation angles $\theta_{1} \ldots \theta_{n}$. By re-arranging (2) as $I\left(\theta_{\text {pol }}\right)=I_{+}+I_{-} \cos \left(2 \theta_{\text {pol }}\right) \cos (2 \bar{\theta})+$ $I_{-} \sin \left(2 \theta_{\text {pol }}\right) \sin (2 \bar{\theta})$, we obtain the following (over-constrained) linear system

$$
\left[\begin{array}{ccc}
1 & \cos \left(2 \theta_{1}\right) & \sin \left(2 \theta_{1}\right) \\
\vdots & & \\
1 & \cos \left(2 \theta_{n}\right) & \sin \left(2 \theta_{n}\right)
\end{array}\right] \mathbf{X}=\left[\begin{array}{c}
I\left(\theta_{1}\right) \\
\vdots \\
I\left(\theta_{n}\right)
\end{array}\right]
$$



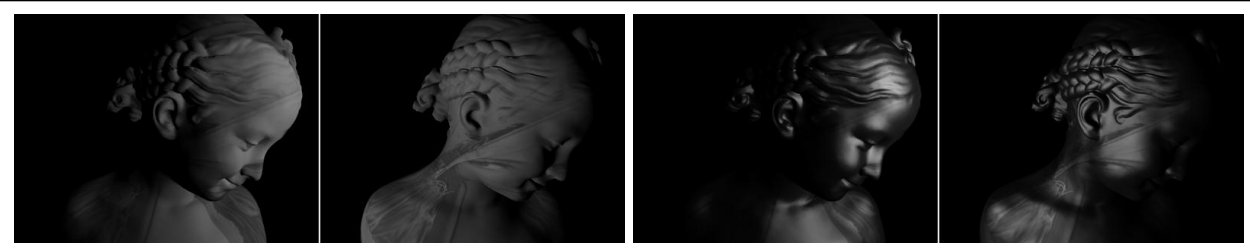

Figure 1: Photometric stereo pair images. left Lambertian, right Cook \& Torrance Specular.

With $\mathbf{X}=\left[X^{1}, X^{2}, X^{3}\right]^{t}=\left[I_{+}, I_{-} \cos (2 \bar{\theta}), I_{-} \sin (2 \bar{\theta})\right]^{t}$. We solve (19) using $L_{1}$ relaxation [ᄆ] and calculate $I_{-}=\left\|\left(X^{2}+X^{3}\right)\right\|_{2}$ and $\bar{\theta}=\operatorname{atan} 2\left(X^{2}, X^{3}\right)$. Finally, $I_{0}$, and $I_{\frac{\pi}{4}}$ are recalculated using (2) with the robustly estimated sinusoidal parameters and used to find the level-set with (11).

The polarisation equation (11) is stacked along equation (16) giving the following variational problem

$$
\min _{z}\left\|\left[\begin{array}{c}
\mathbf{b}_{p s} \\
\mathbf{b}_{p o l}
\end{array}\right] \cdot \nabla z-\left[\begin{array}{c}
s_{p s} \\
0
\end{array}\right]\right\|_{L^{2}}+\lambda\left\|z-z_{0}\right\|_{L^{2}}
$$

where $\lambda=10^{-5}$ in the experiments.

Note that the term $\lambda\left\|z-z_{0}\right\|_{L^{2}}$ is a zero-order Tikhonov regularizer that constraints the mean depth and ensures that the differential problem has a unique solution.

Equation (20) is discretized with finite differences and solved with simple least squares. Furthermore, since $\mathbf{b}_{p s}$ and $s_{p s}$ (but not $\mathbf{b}_{p o l}$ ) implicitly depend on $z$, the solution of Equation (20) is embedded in an iterative process that calculates all relevant quantities $\left(a, \mathbf{h}, \mathbf{b}_{p s}\right.$ and $s_{p s}$ ) by using the current estimates of the depth values in a similar manner with [ $\left.\square\right]$ ]. The optimisation is initialised with a flat plane at the mean distance (measured with a ruler).
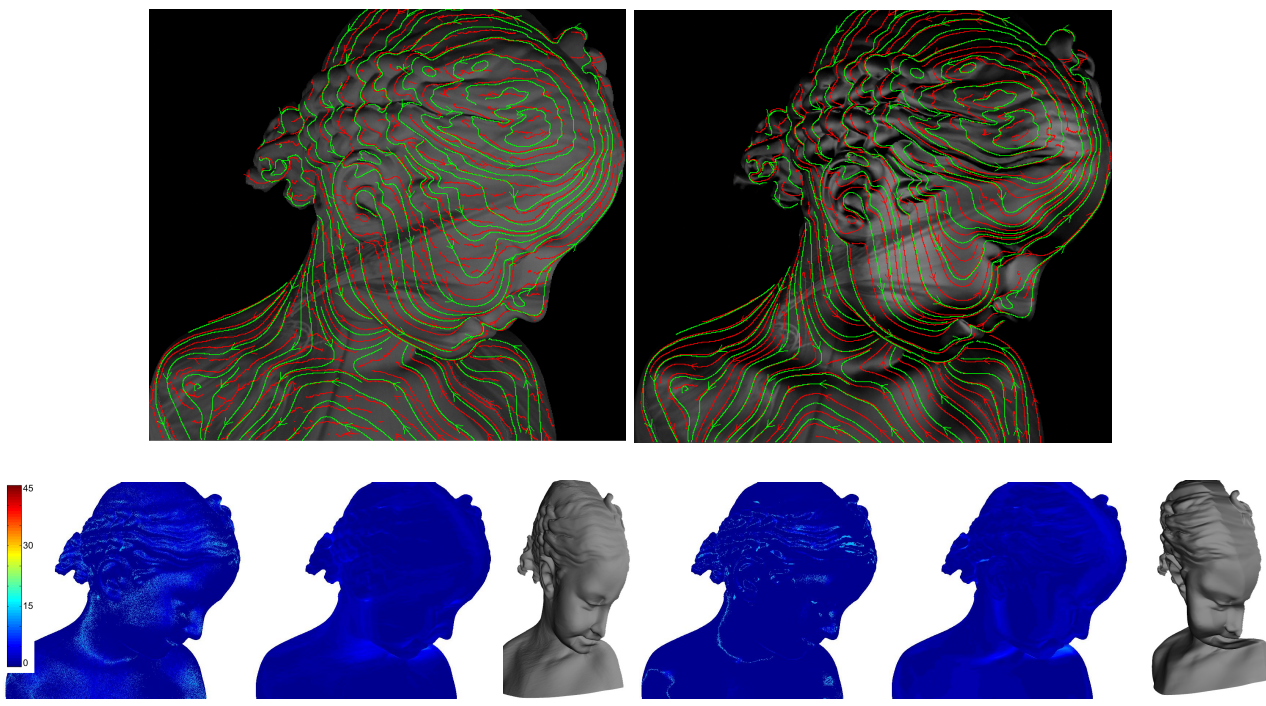

Figure 2: Top row: Bimba level sets for diffuse (left) and specular (right). The ground truth is shown in green, the calculated in red. Bottom row: respective errormaps (for level sets and final normals) and reconstructions. The mean errors are $8.7^{\circ}$ and $12.0^{\circ}$ for the diffuse and $2.4^{\circ}$ and $14.5^{\circ}$ for the specular cases respectively. 

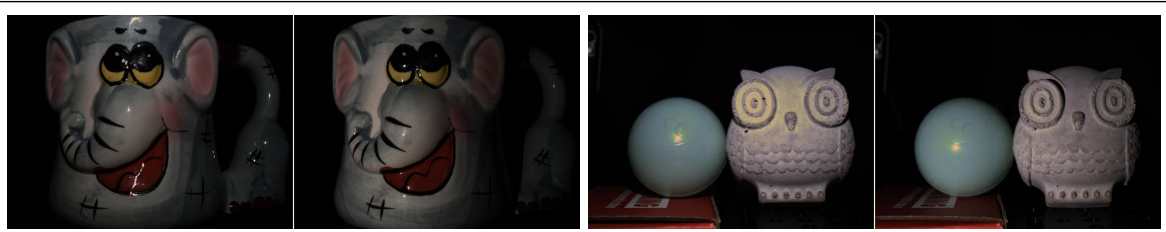

Figure 3: Real data photometric stereo pairs.

\section{Experimental Results}

The proposed approach was evaluated with a range of synthetic and real data sets. In addition, we calculated the level set of the head dataset from [ $\square]$ ]. The algorithm was implemented in matlab with a total running time of about two minutes (for the $7 \times 3 \mathrm{MPixel}$ images) on a laptop with a quad, core-i7, $2.6 \mathrm{GHz} \mathrm{CPU}$.

It is worth mentioning that this experimental section is conceived to validate the theoretical concepts of Section 3, where isocontours of the surface have been described through the proposed differential model (11). Furthermore, 3D reconstruction using SfP and SfPS as described in Section 4 are provided as proof of concept using the minimal amount of data, i.e. without aiming at reconstructing highly accurate shapes.

\subsection{Synthetic Data}

First of all, we generated 800x600 pixel synthetic data using the "bimba" from the AIM@ Shape Repository. The data were rendered with realistic effects including non-uniform albedo, perspective viewing geometry, and near point light sources. We generated a Lambertian and a specular dataset, the latter rendered with the Cook \& Torrance (C\&T) BRDF (see Figure 1).

We rendered the minimum required polarisation images $I_{0}, I_{\frac{\pi}{4}}$ and $I_{\frac{\pi}{2}}$ generated from (2) and assuming index of refraction $\mu=1.6$. Finally, to make the experiments realistic, we limited the precision of the data to 3 decimal digits ${ }^{1}$ and added $0.5 \%$ Gaussian noise.

The level sets obtained are evaluated quantitatively by calculating the angle at each pixel of the computed level set with the ground truth. For reconstructing the specular dataset, we used $c=0.25$ (see (13)). The results are shown in Figure 2. We note that the specular dataset outperforms the diffuse one on the level-set accuracy ( $2.4^{\circ}$ vs $8.7^{\circ}$ mean error), due to the much higher polarisation effects for specular materials. The reconstruction is marginally worse $\left(14.5^{\circ} \text { vs } 12.0^{\circ} \text { mean error }\right)^{2}$ as equation 13 only approximates the C\&T BRDF.
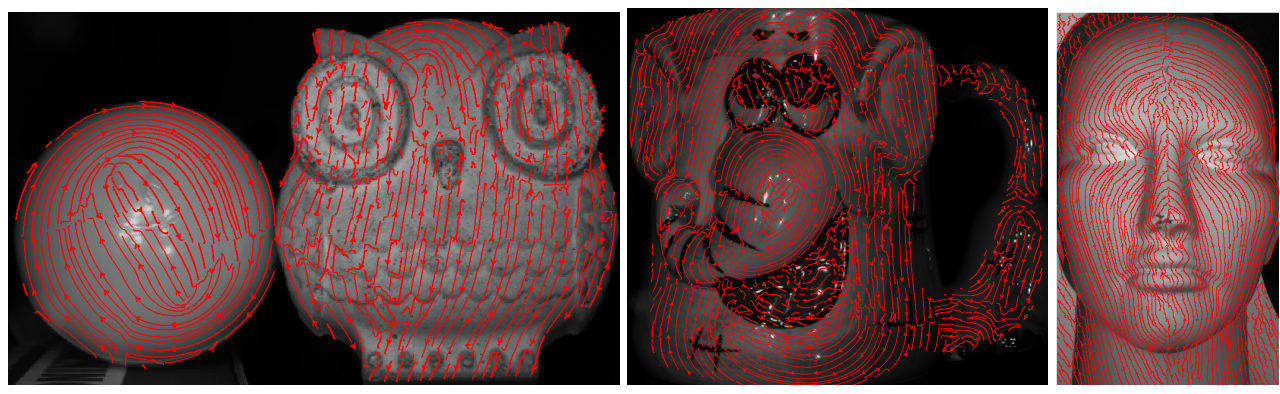

Figure 4: Real data level-sets overlaid on $I_{0}$. Left and middle our data, right [ $\left.\square\right]$.

\footnotetext{
${ }^{1}$ standard cameras offer $10 \mathrm{bit}$ precision raw data.

${ }^{2}$ Reconstructions are evaluated by finding the normals (by differentiation) and comparing to ground truth.
} 


\subsection{Real Data}

The setup we used for acquiring data suitable for SfP and SfPS consists of a Point Grey camera FL3-U3-32S2C-CS having maximum resolution 2080 x 1552 mounting a TECHSPEC $8 \mathrm{~mm}$ UC series fixed focal length lens, OSRAM Platinum Dragon high power LEDs white and a linear polariser mounted on a rotary mount with post. We captured 7 polarisation images at angles $\left[\begin{array}{lllllll}-90 & -60 & -30 & 0 & 30 & 60 & 90\end{array}\right]^{\circ}$.

Our real data sets include a porcelain cup, a plastic ball and ceramic statue as shown in Figure 3. The obtained isocontorus and reconstructions are shown in Figures 4 and 5 respectively. We note that real data experiments confirm the fact that the level sets are more accurately calculated on specular than diffuse materials which is a clear advantage of the SfP approach compared to SfPS.

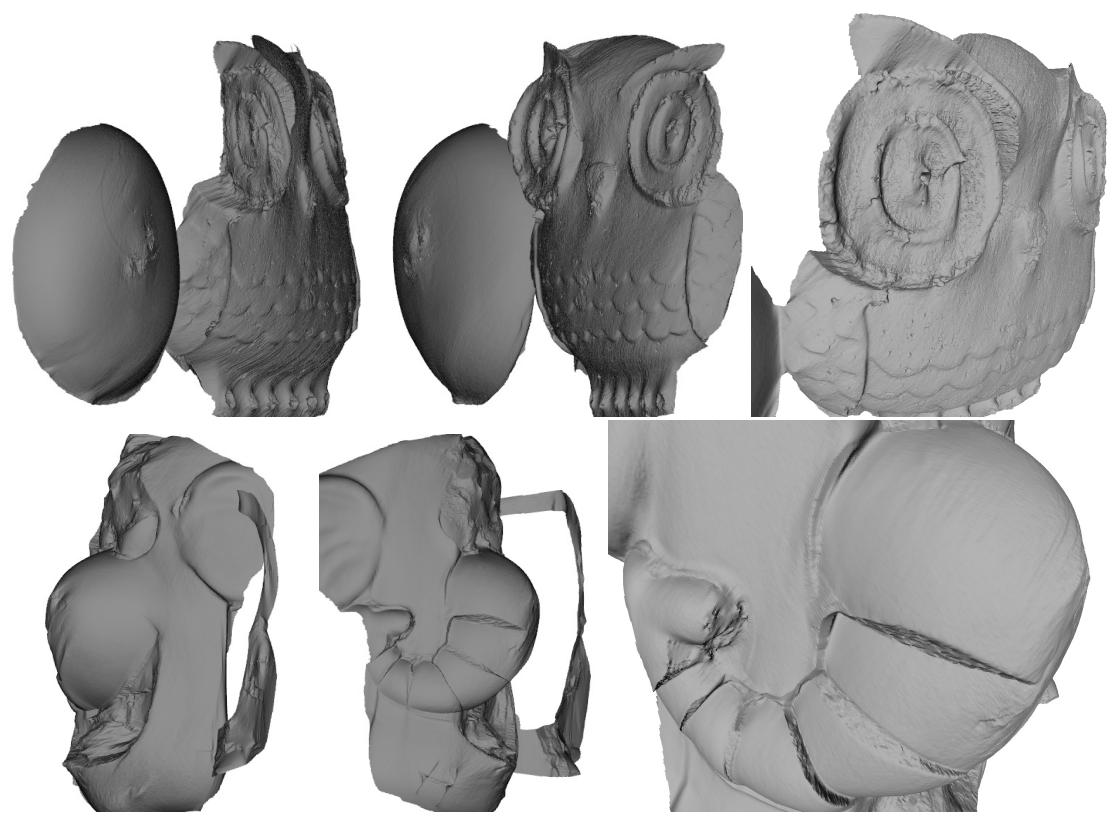

Figure 5: Several views of the reconstructions obtained by fusing the Photometric stereo pairs of Figure 3 and the isocontours of Figure 4.

\section{Conclusion and Perspective}

In this work we presented a first attempt at describing the Shape from Polarisation problem with an albedo and diffuse/specular reflection independent differential formulation. By combining surface depth related parameters into the polarisation image formation, we derived an homogeneous linear PDE that describes the geometry of the surface through its isocontours.

With the aim of providing full 3D shape recovery, we added shading cues using accurate Photometric Stereo model describing sharing information from a point light source with perspective deformation and diffuse/specular reflection. We showed that the new Shape from Polarisation differential formulation merges very elegantly into a system of hyperbolic PDEs which is albedo independent and well-posed by considering two lights sources at least.

This new Shape from Polarisation modelling is open to a number of interesting extensions of the method including merging with multi-view or Shape from Defocusing data. 


\section{References}

[1] G. A. Atkinson and E. R. Hancock. Multi-view surface reconstruction using polarization. In International Conference on Computer Vision (ICCV), 2005.

[2] G. A. Atkinson and E. R. Hancock. Recovery of surface orientation from diffuse polarization. IEEE Transactions on Image Processing, 15(6):1653-1664, 2006.

[3] G. A. Atkinson and E. R. Hancock. Polarization-based surface reconstruction via patch matching. In Conference on Computer Vision and Pattern Recognition (CVPR), 2006.

[4] G. A. Atkinson and E. R. Hancock. Shape estimation using polarization and shading from two views. IEEE Transactions on Pattern Analysis and Machine Intelligence (PAMI), 29(11):2001-2017, 2007.

[5] G. A. Atkinson and E. R. Hancock. Surface reconstruction using polarization and photometric stereo. In Computer Analysis of Images and Patterns (CAIP), 2007.

[6] A. M. Bruckstein. On shape from shading. Computer Vision, Graphic, and Image Processing, 44(2):139-154, 1988.

[7] E. J. Candès, M. B. Wakin, and S. P. Boyd. Enhancing sparsity by reweighted $l_{1}$ minimization. Journal of Fourier Analysis and Applications, 14(5):877-905, 2008.

[8] M. Chandraker, J. Bai, and R. Ramamoorthi. On Differential Photometric Reconstruction for Unknown, Isotropic BRDFs. IEEE Transactions on Pattern Analysis and Machine Intelligence (PAMI), 35(12):2941-2955, 2013.

[9] T. Chen, H. P. A. Lensch, C. Fuchs, and H. P. Seidel. Polarization and phase-shifting for $3 \mathrm{~d}$ scanning of translucent objects. In Conference on Computer Vision and Pattern Recognition (CVPR), 2007.

[10] P. A. Davis and L. A. Soderblom. Modeling crater topography and albedo from monoscopic viking orbiter images: 1. methodology. Journal of Geophysical Research: Solid Earth, 89(B11):9449-9457, 1984.

[11] O. Drbohlav and R. Sara. Unambiguous determination of shape from photometric stereo with unknown light sources. In International Conference on Computer Vision (ICCV), 2001.

[12] C. P. Huynh, A. Robles-Kelly, and E. R. Hancock. Shape and refractive index from single-view spectro-polarimetric images. International Journal of Computer Vision (IJCV), 101(1):64-94, 2013.

[13] A. Kadambi, V. Taamazyan, B. Shi, and R. Raskar. Polarized 3d: High-quality depth sensing with polarization cues. In International Conference on Computer Vision (ICCV), 2015.

[14] K. Koshikawa. A polarimetric approach to shape understanding of glossy objects. In International Joint Conference on Artificial Intelligence (IJCAI), pages 493-495, 1979.

[15] F. Logothetis, R. Mecca, Y. Quéau, and R. Cipolla. Near-field photometric stereo in ambient light. In British Machine Vision Conference (BMVC), 2016. 
[16] F. Logothetis, R. Mecca, and R. Cipolla. Semi-calibrated near field photometric stereo. Computer Vision and Pattern Recognition (CVPR), 2017.

[17] R. Martinez-Herrero, P. M. Mejias, and G. Piquero. Characterization of Partially Polarized Light Fields. Springer-Verlag Berlin Heidelberg, 2009.

[18] R. Mecca and M. Falcone. Uniqueness and approximation of a photometric shapefrom-shading model. SIAM Journal on Imaging Sciences, 6(1):616-659, 2013.

[19] R. Mecca, A. Tankus, A. Wetzler, and A. M. Bruckstein. A Direct Differential Approach to Photometric Stereo with Perspective Viewing. SIAM Journal on Imaging Sciences, 7(2):579-612, 2014.

[20] R. Mecca, A. Wetzler, A. Bruckstein, and R. Kimmel. Near Field Photometric Stereo with Point Light Sources. SIAM Journal on Imaging Sciences, 7(4):2732-2770, 2014.

[21] R. Mecca, E. Rodolà, and D. Cremers. Realistic photometric stereo using partial differential irradiance equation ratios. Computers \& Graphics, 51:8-16, 2015.

[22] R. Mecca, Y. Quéau, F. Logothetis, and R. Cipolla. A single lobe photometric stereo approach for heterogeneous material. SIAM Journal on Imaging Sciences, 9(4):1858$1888,2016$.

[23] D. Miyazaki and K. Ikeuchi. Inverse polarization raytracing: estimating surface shapes of transparent objects. In Conference on Computer Vision and Pattern Recognition (CVPR), 2005.

[24] D. Miyazaki and K. Ikeuchi. Shape estimation of transparent objects by using inverse polarization ray tracing. IEEE Transactions on Pattern Analysis and Machine Intelligence (PAMI), 29(11):2018-2030, 2007.

[25] D. Miyazaki, M. Kagesawa, and K. Ikeuchi. Polarization-based transparent surface modeling from two views. In International Conference on Computer Vision (ICCV), 2003.

[26] D. Miyazaki, M. Kagesawa, and K. Ikeuchi. Transparent surface modeling from a pair of polarization images. IEEE Transactions on Pattern Analysis and Machine Intelligence (PAMI), 26(1):73-82, 2004.

[27] D. Miyazaki, T. Shigetomi, M. Baba, R. Furukawa, S. Hiura, and N. Asada. Polarization-based surface normal estimation of black specular objects from multiple viewpoints. In Conference on 3D Imaging, Modeling, Processing, Visualization Transmission (3DIMPVT), 2012.

[28] O. Morel, M. Ferraton, C. Stolz, and P. Gorria. Active lighting applied to shape from polarization. In International Conference on Image Processing (ICIP), 2006.

[29] S. K. Nayar, X. S. Fang, and T. Boult. Removal of specularities using color and polarization. In Conference on Computer Vision and Pattern Recognition (CVPR), 1993.

[30] T. T. Ngo, H. Nagahara, and R. I. Taniguchi. Shape and light directions from shading and polarization. In Conference on Computer Vision and Pattern Recognition (CVPR), 2015. 
[31] W. A. P. and F. Fang. Height from photometric ratio with model-based light source selection. Computer Vision and Image Understanding, 145:128-138, 42016.

[32] T. Papadhimitri and P. Favaro. A New Perspective on Uncalibrated Photometric Stereo. In Conference on Computer Vision and Pattern Recognition (CVPR), 2013.

[33] E. Prados and O. D. Faugeras. Shape from shading: A well-posed problem? In Conference on Computer Vision and Pattern Recognition (CVPR), 2005.

[34] Y. Quéau, R. Mecca, J.-D. Durou, and X. Descombes. Photometric stereo with only two images: A theoretical study and numerical resolution. Image Vision Comput., 57: 175-191, 2017.

[35] S. Rahmann and N. Canterakis. Reconstruction of specular surfaces using polarization imaging. In Conference on Computer Vision and Pattern Recognition (CVPR), 2001.

[36] M. Saito, Y. Sato, K. Ikeuchi, and H. Kashiwagi. Measurement of surface orientations of transparent objects using polarization in highlight. In Conference on Computer Vision and Pattern Recognition (CVPR), 1999.

[37] W. A. P. Smith, R. Ramamoorthi, and S. Tozza. Linear depth estimation from an uncalibrated, monocular polarisation image. In European Conference on Computer Vision (ECCV), 2016.

[38] A. Tankus, N. A. Sochen, and Y. Yeshurun. A new perspective [on] shape-fromshading. In International Conference on Computer Vision (ICCV), 2003.

[39] K. E. Torrance and E. M. Sparrow. Theory for off-specular reflection from roughened surfaces. J. Opt. Soc. Am., 57(9):1105-1114, Sep 1967.

[40] S. Umeyama and G. Godin. Separation of diffuse and specular components of surface reflection by use of polarization and statistical analysis of images. IEEE Transactions on Pattern Analysis and Machine Intelligence (PAMI), 26(5):639-647, 2004.

[41] F. Wang, S. Ainouz, C. Petitjean, and A. Bensrhair. Polarization-based specularity removal method with global energy minimization. In International Conference on Image Processing (ICIP), 2016.

[42] L. B. Wolff. Scene understanding from propagation and consistency of polarizationbased constraints. In Conference on Computer Vision and Pattern Recognition (CVPR), 1994.

[43] L. B. Wolff. Polarization camera for computer vision with a beam splitter. Journal of the Optical Society of America A, 11:2935-2945, November 1994.

[44] L. B. Wolff and T. E. Boult. Constraining object features using a polarization reflectance model. IEEE Transactions on Pattern Analysis and Machine Intelligence (PAMI), 13(7):635-657, 1991.

[45] Q. Zhu and J. Shi. Shape from shading: Recognizing the mountains through a global view. In Conference on Computer Vision and Pattern Recognition (CVPR), 2006. 